\title{
Esophageal Adenocarcinoma by AJCC v8 Postneoadjuvant Therapy Stage
}

National Cancer Institute

\section{Source}

National Cancer Institute. Esophageal Adenocarcinoma by A/CC v8 Postneoadjuvant

Therapy Stage. NCI Thesaurus. Code C133432.

A term that refers to the postneoadjuvant therapy staging of esophageal

adenocarcinoma according to the American Joint Committee on Cancer, 8th edition. 